\title{
14. On the Hypoglycemic Action of Oxalic Acid. (2nd Report.)
}

\author{
By Taizo KumagaI, M.J.A., Hideo Endo, Kenji KumagaI, \\ and Yosio OIKawa. \\ Institute of Tuberculosis and Leprosy, Tohoku University, Sendai. \\ (Comm. by T. KumagaI, M.S.A., June 12, 1948.)
}

In the previous report ${ }^{1)}$ we have reasoned out a conclusion that there must be two kinds of oxalic acid, physiologically active and inactive. The reason why: (1) water extract of "Renriso", Lathyrus palustris $L$. var macranthus (White) has a remarkable therapeutic effect for diabetes mellitus. (2) An effectual substance, which has the same chemical and physical properties as oxalic acid was presented from "Renriso". (3) We ascertained that 1-2 mg of this oxalic acid-like substance would kill 1-1.5 $\mathrm{kg}$ weight rabbits within 45 hrs. with hypoglycemic convulsion. (4) On the other hand oxalic acids for sale and more than $100 \mathrm{mg}$ of this acid kills rabbits of $1-1.5 \mathrm{~kg}$ weight.

Rabbits die under hypoglycemic convulsion by the injection of this active oxalic acid as in the case of Insulin injection. According to many author's experiments a toxicosis of oxalic acid is thought to be caused by the removal of calcium from the tissues and from the blood. Therefore we have measured calcium content in blood of poisoned rabbits. For the determination of calcium the method of Clark-Collip (J. Biol. Chem., 63, 1925) was adopted. It was observed that the amount of calcium in blood serum of five normal healthy rabbits is within from 9 to $15 \mathrm{mg}$. per 100 cc. By subcutaneous administration of about $200 \mathrm{mg}$., of oxalic acid Merk, calcium content of blood serum is in the minimum normal value, but $500 \mathrm{mg}$. of this acid reduces calcium content to $2.8 \mathrm{mg}$ per $100 \mathrm{cc}$. Compared with the control, decrease of calcium content is not remarkable when $1 \mathrm{~g}$ of the sample has been given to a rabbit of $3.7 \mathrm{~kg}$ weight. Soon after the hypoglycemic convulsion, caused by injection of 1 to $5 \mathrm{mg}$ oxalic acid, which was prepared from "Renriso" and Rumex japonicus Meisn. The amount of calcium of blood serum was measured

1) Vol. 21, No. 9 of the Proc. 


\begin{tabular}{|c|c|c|c|c|c|c|c|}
\hline \multicolumn{3}{|c|}{ 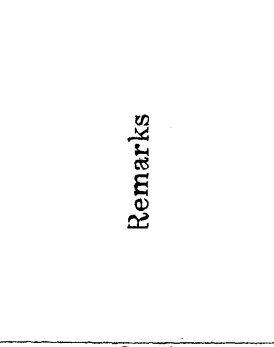 } & 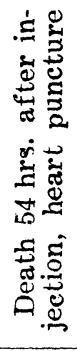 & 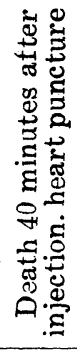 & 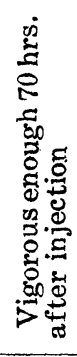 & 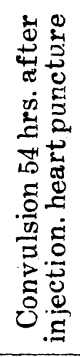 & 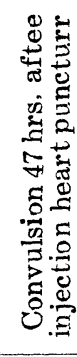 \\
\hline \multirow{12}{*}{ 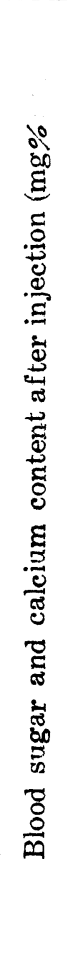 } & \multirow{2}{*}{ 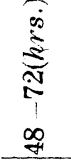 } & $\tilde{U}$ & 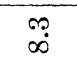 & & $\begin{array}{l}0 \\
0\end{array}$ & $\begin{array}{l}\stackrel{9}{0} \\
\stackrel{10}{\rightarrow}\end{array}$ & \\
\hline & & $\begin{array}{l}\square \\
8 \\
0.0 \\
0.0 \\
0\end{array}$ & $\mathscr{9}$ & & 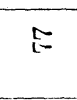 & ๙ & \\
\hline & \multirow{2}{*}{$\begin{array}{l}\infty \\
\stackrel{\infty}{1} \\
\stackrel{N}{+}\end{array}$} & $\widetilde{0}$ & & & $\stackrel{\leftrightarrow}{\stackrel{\oplus}{\oplus}}$ & & $\stackrel{\check{I}}{\stackrel{\Xi}{N}}$ \\
\hline & & $\begin{array}{l}2 \\
0 \\
0 \\
0 \\
0\end{array}$ & $\stackrel{19}{\cong}$ & & $\Xi$ & $\stackrel{\circ}{\sim}$ & $\stackrel{\bullet}{\sim}$ \\
\hline & \multirow{2}{*}{$\begin{array}{c}\stackrel{\sim}{N} \\
\stackrel{\sim}{\sim}\end{array}$} & రే & & & $\overrightarrow{0}$ & & \\
\hline & & $\begin{array}{l}0.0 \\
80 \\
0.0 \\
0.0\end{array}$ & $\stackrel{\mathscr{I}}{\mathbb{Z}}$ & & $\stackrel{\mathscr{g}}{-1}$ & $\frac{10}{10}$ & $\stackrel{\infty}{\infty}$ \\
\hline & \multirow{2}{*}{$\stackrel{0}{7}$} & $\tilde{J}$ & & & & & \\
\hline & & 要 & 荥 & & & & 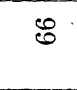 \\
\hline & \multirow{2}{*}{$\begin{array}{l}0 \\
1 \\
0\end{array}$} & تే & & $\begin{array}{l}\infty \\
\stackrel{\infty}{N}\end{array}$ & $\overrightarrow{0}$ & & \\
\hline & & 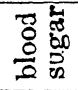 & 节 & D्ञ & ్ㅗ & $\overrightarrow{0}$ & $\stackrel{\mathbb{E}}{\mathbb{2}}$ \\
\hline & \multirow{2}{*}{ 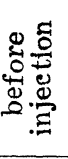 } & $\tilde{0}$ & & & $\stackrel{\ddot{J}}{\stackrel{H}{+}}$ & & \\
\hline & & $\begin{array}{l}8 \\
80 \\
05 \\
0\end{array}$ & $\stackrel{\varrho}{=}$ & $\overrightarrow{\stackrel{9}{9}}$ & త్తి & 8 & $\infty$ \\
\hline \multicolumn{3}{|c|}{$\begin{array}{l}\text { (.8u) uo!̣exłs! } \\
\text {-u!upe Jo łunouiv }\end{array}$} & 오 & 8 & 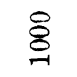 & - & 20 \\
\hline \multirow{2}{*}{$\frac{v}{5}$} & & $\sum^{\frac{n}{0}}$ & $\ddot{8}$ & ్ㅗㅇ & $\underset{2}{2}$ & 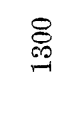 & $\begin{array}{l}8 \\
\stackrel{\circ}{-1}\end{array}$ \\
\hline & & $\begin{array}{c}\dot{\Phi} \\
\frac{\tilde{D}}{\tilde{\Xi}} \\
z\end{array}$ & $\begin{array}{l}\stackrel{0}{\cdots} \\
\dot{4}\end{array}$ & $\stackrel{2}{\stackrel{\leftrightarrow}{4}}$ & $\stackrel{9}{\stackrel{4}{4}}$ & 한 & $\begin{array}{l}\infty \\
\stackrel{4}{4}\end{array}$ \\
\hline \multicolumn{3}{|c|}{ 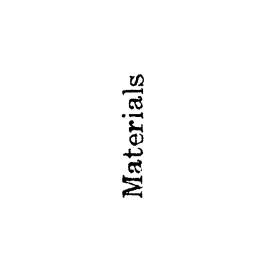 } & 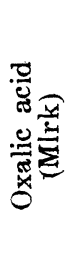 & 8 & 8 & 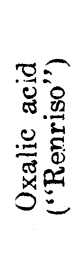 & 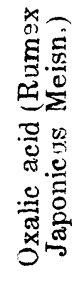 \\
\hline
\end{tabular}


and we have known the calcium content within normal value (Table I). There is no doubt on the ground that convulsed rabbits have recovered from poisoning by injection of grape sugar.

Oxalic acid is distributed universally over the vegetable world. But its physiological meaning is not clear. According to many authors oxalic acid is perhaps an excreta of metabolism. Some authors think that it may be an intermediate product of metabolism. It is known that in the human being and animals oxalic acid is excreted in their urine. To examine the action of oxalic acid in urine we have separated this acid in urine by the following process : 100 litre urine has been gathered, making a weak alkaline solution by adding ammonia and calcium chloride. The precipitate has been filtered and washed with water several times and by adding acetic acid the soluble salte were dissolved and the solution has been extracted with ether in Soxhletapparatus for $48 \mathrm{hrs}$; after evaporation of ether oxalic acid has been separated from other mixed substances. $1 \mathrm{mg}$ of this purified substance kills similarly rabbits under hypoglycemic convulsion as with oxalic acid from "Renriso". (Table II.)

Furthermore we prepared oxalic acid from tea-leaves, woodsorrel, sorrel-dock, Rumex japonicus Meisn. Polygonum Reynoutria Makino and several moulds. And we ascetained that their pharmacological actio 1 and toxic symptoms are the same as oxalic acid from "Renriso". In the way we have known that there is in nature many oxalic acid of same physiological action as the one from "Renriso". 


\begin{tabular}{|c|c|c|c|c|c|c|c|c|c|c|c|}
\hline \multicolumn{2}{|c|}{ 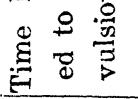 } & $N \pi$ & 520 & స్ర0 & Nis & N & 74 & ลีลง & $\Delta F$ & 6 & tr \\
\hline \multirow{2}{*}{ रे } & $\overbrace{10}^{N}$ & & 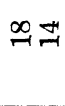 & $8 \%$ & & & & 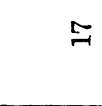 & & 心゙ & \\
\hline & $\begin{array}{r}10 \\
10 \\
1 \\
+1 \\
\end{array}$ & & $5 \%$ & 온닥 & 学 & $\stackrel{\infty}{\stackrel{N}{N}}$ & L & เి & & $\overleftarrow{ล}$ & ন \\
\hline $\begin{array}{l}\text { है } \\
\text { हี } \\
.0\end{array}$ & $\begin{array}{l}\infty \\
1 \\
0 \\
0\end{array}$ & & 용 & 87 & $\stackrel{ }{\sim}$ & 尺 & $\Leftrightarrow$ & s & $\overleftarrow{ง}$ & ๗ิ & เง \\
\hline 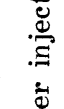 & $\begin{array}{l}8 \\
1 \\
\text { N } \\
\end{array}$ & คิ & ळ喵 & สతి\& & હ゙こ & $\infty$ & 事里 & $\stackrel{\infty}{\infty} \stackrel{\infty}{+\infty}$ & 80 & $\stackrel{\infty}{q}$ & 요 \\
\hline 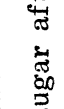 & 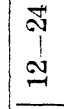 & คిন & 80 & 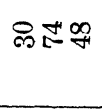 & 这造 & $9 \overrightarrow{+}$ & $\mathscr{P}_{7}^{\infty}$ & 푼 & 옹요 & $\infty$ & \& \\
\hline$\frac{3}{2}$ & $\stackrel{?}{?}$ & & 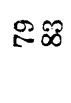 & $\infty$ & $\stackrel{10}{2}$ & $\stackrel{\infty}{\infty}$ & $\infty$ & $\stackrel{20}{\circ}$ & $\stackrel{\infty}{1}$ & $\infty$ & $\bar{\infty}$ \\
\hline$\stackrel{\overbrace{}}{\stackrel{0}{*}}$ & $\begin{array}{l}0 \\
\vdots \\
\text { is }\end{array}$ & 100 & & $\stackrel{\infty}{\infty} \underset{i}{ }$ & 120 & $\mathscr{\infty}$ & & $\infty \infty_{\infty}^{\infty}$ & $\stackrel{\infty}{\infty}$ & 5 & $\stackrel{\circ}{0}$ \\
\hline$>$ & p & & $\llcorner\infty$ & ํํㅇ & $\infty$ & 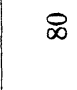 & 때 & 8 & & & \\
\hline & 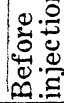 & 102 & $\tilde{\Sigma}^{2}$ & $\check{\infty} \infty$ & $\infty \vec{\infty}$ & $\infty \infty$ & ஜ கి & $\stackrel{\infty}{\infty}$ ఠ゙ & 음요 & $\frac{10}{5}$ & $\vec{\infty}$ \\
\hline
\end{tabular}

(siu) uongex

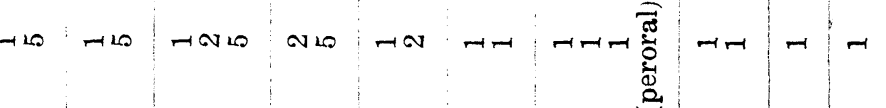
-7stutupe

\begin{tabular}{|c|c|c|c|c|c|c|c|c|c|c|c|}
\hline \multicolumn{4}{|c|}{ r } & & & & & \multicolumn{2}{|c|}{2} & \multirow[b]{2}{*}{ 品 } & \multirow[b]{2}{*}{ 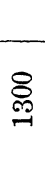 } \\
\hline \multirow{2}{*}{ 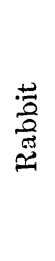 } & $\underbrace{80}$ & 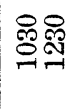 & 웅요 & ํํำ & 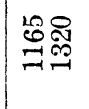 & స్ి유 & 骨? & ి్రిల్రి & 욬웜 & & \\
\hline & 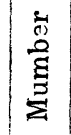 & 齊员 & ํㅐㅁㅇㅛ & 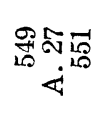 & 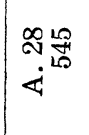 & 舟 & 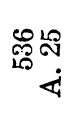 & 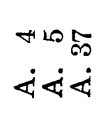 & 或 & $\overrightarrow{4}$ & $\begin{array}{l}\infty \\
\dot{4}\end{array}$ \\
\hline & $\overrightarrow{0}$ & 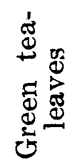 & 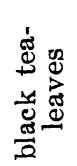 & 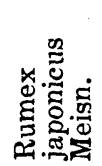 & 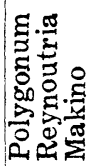 & 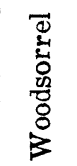 & 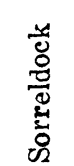 & 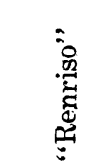 & 竞 & 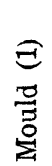 & 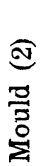 \\
\hline
\end{tabular}

